\title{
3D hydrodynamical simulations of corotating interaction regions in rotating line-driven stellar winds
}

\author{
L. Dessart
}

\author{
Max-Planck-Institut für Astrophysik,Karl-Schwarzschild-Straße 1, 85748 Garching bei Muenchen, Germany \\ e-mail: luc@mpa-garching.mpg.de
}

Received 29 March 2004 / Accepted 4 May 2004

\begin{abstract}
I present radiation hydrodynamics simulations of rotating line-driven O-star winds subject to surface variations taking the shape of localized bright Gaussian spots. Following the original work of Cranmer \& Owocki, I investigate the influence on resulting corotating interaction regions (CIRs) of 1) extending the simulation domain from 2D (equatorial plane) to 3D (octant of a sphere) and 2) explicitly account for the lateral components of the radiative acceleration, computed using a multiple-ray quadrature of the visible stellar disk at all wind locations. I identify the wind spin down effect of Gayley \& Owocki, present in unperturbed rotating hot star winds. In 2D simulations, the perturbed azimuthal component of the radiative acceleration does not change the gross properties of CIRs. However, the resulting perturbed azimuthal velocity extrema are enhanced by a factor of 50 compared to unperturbed models, so that its magnitude is now a few times greater than the adopted isothermal sound speed. This lateral broadening of wind material at the vertical of a spot leads to an overall weakening of the CIR compression compared to Cranmer \& Owocki. In 3D, the extra dimension weakens further the CIR compression and associated velocity kink compared to equivalent 2D simulations. 3D simulations confirm the assumption of Cranmer \& Owocki that the angular extent of a surface spot influence is similar in the azimuthal and latitudinal directions. 3D simulations for off-equatorial spots reveal the presence of CIRs advecting out along a fixed latitude, i.e. centred on the spot latitude and contained within a conic shell whose latitudinal thickness is of the order of the full-width-half-maximum of the Gaussian spot. Thus, the CIR properties are essentially independent of the latitude of base perturbations. These results suggest that the key properties of a line-driven wind subject to base brightness variations are well described by considering only the perturbation on the radial radiative acceleration, with, for a Gaussian spot, an angular modulation solely dependent on the distance to the spot location, irrespective of the latitudinal or azimuthal direction. Following the technique of Cranmer \& Owocki, I have performed spectroscopic line synthesis computations based on 2D hydrodynamical inputs obtained using the multi-ray computation of the multi-component perturbed radiative acceleration or its perturbed radial component. For both P-Cygni and emission line profiles, the differences in hydrodynamical inputs have unnoticeable effects on profile shapes. The method developed in Cranmer \& Owocki is therefore adequate for extensive investigations of the large-amplitude long-term line profile variability identified in many O-star spectra.
\end{abstract}

Key words. hydrodynamics - stars: early-type - stars: mass-loss

\section{Introduction}

Hot and luminous stars are characterized by fast $\left(v_{\infty} \sim\right.$ $\left.1000 \mathrm{~km} \mathrm{~s}^{-1}\right)$ and dense $\left(\dot{M} \sim 10^{-6} M_{\odot} \mathrm{yr}^{-1}\right)$ outflows stemming from the large radiation pressure on optically thick lines of metals (Castor et al. 1975; Lucy \& Solomon 1970). Although these mass loss properties are for many objects essentially constant, some exhibit a noticeable time-variability. For few exceptional cases of supposedly single objects, this timevariability is seen photometrically (e.g. for Luminous Blue Variables, Humphreys \& Davidson 1994). But in general, it is observed spectroscopically in the absorption troughs of UV PCygni profiles or in the strong optical emission lines of hot star spectra. The general perspective is that such line profile variations are caused by a combination of localized optical depth and emissivity enhancements, associated with temporal changes in the density and velocity properties of these stellar winds.
One must distinguish between two broad categories of line profile variations. First, there exists a small-scale $(\sim 1 \%)$ and short-lived ( $\sim$ hours) spectroscopic variability identified in high $\mathrm{S} / \mathrm{N}$ observations of $\mathrm{O}$ and WR stars (Eversberg et al. 1998; Robert 1992; Lépine \& Moffat 1999). These variations are associated with the presence of a large number of radially and laterally confined density enhancements (clumps) distributed randomly throughout the wind and representing most of its mass. They are believed to stem from the intrinsic instability of line-driving (Owocki \& Rybicki 1984; Owocki et al. 1988) which provides a satisfactory explanation for the observed line profile variations (Dessart \& Owocki 2002a,b, 2003). Secondly, and this will be the general focus of the present work, another type of spectroscopic variability has been extensively observed with IUE (Prinja et al. 1995; Kaper et al. 1997) in UV unsaturated P-Cygni profiles in the form of Discrete Absorption Components (DACs) slowly migrating away from line centre towards its blue edge. This variability 
operates over a longer time scale ( days) and with a much larger amplitude $(\sim 10 \%)$ than the one discussed above. This time scale is in particular much larger than the typical time scale of the wind $\left(R_{\star} / v_{\infty}\right)$, of a few hours. In order for variations to be observable in P-Cygni troughs, these wind structures must have a large enough lateral scale to cover most of the stellar disk as seen by the observer, made possible by the stabilizing effect played by the scattered radiation field in the lateral direction (Rybicki et al. 1990). The flux variations of absorption troughs are sometimes as large as the average flux level itself, suggesting a significant change from the mean wind properties. These properties suggest that the root mechanism is external to the wind, perhaps resulting from complex dynamical effects of a stellar magnetic field, non-radial pulsations, and/or rotation.

Cranmer \& Owocki (1996, hereafter CO) investigated the dynamical impact on a line-driven wind of the presence of brightness perturbations rotating rigidly with the star's photosphere, modifying the fluid properties at the base. Such brightness variations are a convenient way to perturb the outflow at the base but should be seen as an ersatz rather than real entities in a strict sense. As mentioned above, a number of physical mechanisms may cause similar base perturbations for which consistent dynamical modeling is needed. $\mathrm{CO}$ proposed that a variable spectroscopic pattern in $\mathrm{O}$-star winds with a timescale of the order of days would likely result from such brightness variations at the stellar surface. As in the case of the sun (Mullan 1984), CO found, using numerical hydrodynamics simulations based on such a hypothesis, that these brightness perturbations lead to the formation of corotating interaction regions, resulting from the rotational modulation enforced on the wind driving itself, with azimuthally modulated mass loss properties. CO and later Owocki et al. (1997) showed that key features of DACs can be reproduced with this model, through variable optical depth enhancements arising from the combined presence of a plateau in the radial velocity, as well as a density compression.

The numerical investigations of $\mathrm{CO}$ were performed in the equatorial plane of a rotating O-star, e.g. $\zeta$ Puppis. For computational tractability, they were limited to $2 \mathrm{D}$, therefore assuming no latitudinal acceleration or velocity, and forcing spots to be at zero latitude. Here, I generalize their study by doing simulations in 3D, placing spots at any desired latitude. Moreover, they neglected any lateral radiative acceleration, considering in their first approach that the dominant impact is in the radial direction. Assuming a Gaussian spot, their phenomenological approach allowed them to obtain the perturbed radial line-force simply by scaling the unperturbed force, this scaling being determined solely by geometrical considerations and the spot properties. Hence, their method captures the key aspects of the defined problem while remaining computationally cheap. However, one can ask what the dynamical influence of the lateral components of the perturbed line-force would be, especially in the 3D context, and for spots at diverse latitudes. There is no analytical handling of the radiation-force in this case so a numerical ray-quadrature of the stellar disk must be carried out instead.

In the present paper, I present the characteristics of the hydrodynamical scheme used here (Sect. 2.1), the module computing the radiative acceleration (Sect. 2.2) and the resulting unperturbed wind properties, and in particular confirm the wind spin-down effect of Gayley \& Owocki (Sect. 2.3). In Sect. 2.4, I present the way I handle the surface modulation and describe the morphology of the three components of the perturbed radiative acceleration resulting from the presence of four equidistant equatorial spots. In Sect. 3, I present the results of my radiation hydrodynamics simulations for a wide range of assumptions. In Sect. 3.1, I draw the comparison between the relaxed equatorial wind properties for cases where the perturbed radiative acceleration is computed using the formalism of $\mathrm{CO}$, the multi-ray approach in $2 \mathrm{D}$ and $3 \mathrm{D}$, still assuming spots in the equatorial plane. In Sect. 3.2, I present the results for off-equatorial spots. I finally discuss the results of my investigation and summarize the main conclusions in Sect. 4.

\section{Description of the model}

\subsection{The hydro code}

My simulations are carried out with the publicly available hydrodynamical code Zeus3D (Stone \& Norman 1992) using spherical coordinates. This Eulerian code computes the time evolution of a fluid under the constraints of mass and momentum conservation. I assume a uniform temperature, a reasonable approximation given the efficiency of radiative cooling in shocked regions. Away from shocks, the balance between photo-ionization processes and such a radiative cooling maintains the bulk of the material near the effective temperature of the star. Within the basic structure of the code, I implement a radiation force module (Sect. 2.2) and add each component of the computed radiative acceleration in the relevant momentum equation.

For simulations carried out in $2 \mathrm{D}$, the computational domain is the equatorial plane of the rotating $\operatorname{star}(\theta=90 \mathrm{deg})$. In $3 \mathrm{D}$, I limit the latitudinal domain to one hemisphere ( $\theta$ between 0 and $90 \mathrm{deg}$ ), using a reflecting-plane boundary condition at $\theta=90 \mathrm{deg}$ and a reflecting-rotation-axis condition at $\theta=0$. In practice, I only cover a fraction of the full $360 \mathrm{deg}$ azimuthal extent. I use periodic azimuthal boundary conditions and restrict the angular coverage in this direction to $90 \mathrm{deg}$. When I include surface brightness variations, I introduce explicitly only one spot in the simulation domain. Given these boundary conditions, I thus have an effective number of four spots if these are confined to the equatorial plane (spot latitude of zero) and 8 (4 per hemisphere) in 3D simulations with offequatorial spots. This angular grid choice was imposed by the high computational cost of the multi-ray radiation force on a 3D grid (Sect. 2.2.2) and the necessity to compare simulations under similar setups (2D versus $3 \mathrm{D}$ ). The base of the simulation domain is an inflow boundary, for which I set the density at $7 \times 10^{-11} \mathrm{~g} \mathrm{~cm}^{-3}$ and the second radial derivative of the velocity to zero (i.e. constant radial velocity gradient). The latitudinal velocity is forced to zero, while I assume rigid body rotation at the stellar surface, so that all latitudes rotate with the same angular velocity $\Omega$ and $v_{\phi}\left(R_{\star}, \theta, \phi\right)=\Omega R_{\star} \sin \theta$. In the simulations performed, the stellar oblateness resulting from 
the adopted rotation rate is small, so I assume the stellar surface remains exactly spherical, lying at a constant radius $R_{\star}$.

The numerical resolution follows the standard choice for Sobolev simulations of line-driven winds, i.e. where it is not necessary to resolve the Sobolev length. Hence, I use a coarse radial grid, i.e. with only 200 zones, and whose increment increases outward by $3.1 \%$ at every radial index, offering nonetheless a good sampling of the transonic region. To cover well the radial evolution of the perturbed wind, I extend the radial grid from the photosphere out to 30 stellar radii. The latitudinal and azimuthal grids have a uniform resolution, with 60 points to cover $90 \mathrm{deg}$. Note that naturally, this introduces a higher effective resolution at higher latitudes.

\subsection{The radiative acceleration}

At a given wind point $\boldsymbol{r}=(r, \theta, \phi)$, the radiative acceleration $\boldsymbol{g}_{\mathrm{rad}}^{\text {line }}(\boldsymbol{r})$ is obtained by integrating the individual force contributions from the ray-quadrature of the visible stellar disk as seen from $\boldsymbol{r}$ (Eq. (9) of Cranmer \& Owocki 1995),

$$
\begin{aligned}
\boldsymbol{g}_{\mathrm{rad}}^{\text {line }}(\boldsymbol{r})= & \frac{\sigma_{\mathrm{e}}^{1-\alpha} k}{c v_{\mathrm{th}}^{\alpha}} \\
& \times \oint I_{\star}\left(r, \theta^{\prime}, \phi^{\prime}\right)\left(\frac{\boldsymbol{n} \cdot \nabla(\boldsymbol{n} \cdot \boldsymbol{v})}{\rho(r, \theta, \phi)}\right)^{\alpha} \boldsymbol{n} \sin \theta^{\prime} \mathrm{d} \theta^{\prime} \mathrm{d} \phi^{\prime}
\end{aligned}
$$

where $\sigma_{\mathrm{e}}$ is the electron scattering coefficient, $v_{\text {th }}$ a fiducial ion thermal speed, $c$ the speed of light, $\alpha$ and $k$ coefficients characterizing the slope and normalization of the line opacity distribution (Abbott 1982), $I_{\star}$ the specific intensity at $r$ along the direction $\boldsymbol{n}$, described by the observer-centred angles $\theta^{\prime}$ and $\phi^{\prime}$ characterizing the ray quadrature. $\phi^{\prime}$ covers $2 \pi$ but $\theta^{\prime}$ is limited between zero and the stellar limb angle as seen from $\boldsymbol{r}$.

The bolometric intensity arising from the stellar surface is given by

$I_{\star}\left(r, \theta^{\prime}, \phi^{\prime}\right)=\frac{L_{\star}}{4 \pi R_{\star}^{2}} \mathcal{D}\left(r, \mu^{\prime}\right)$

where $L_{\star}$ and $R_{\star}$ are the stellar luminosity and radius, $\mathcal{D}\left(r, \mu^{\prime}\right)$ is the limb darkening law and the $\mu^{\prime} \equiv \cos \theta^{\prime}$.

Assuming a uniformly bright disk is mathematically convenient since in that case, for a purely radial expansion, the above expression can be evaluated analytically. I describe in the next section this approach, followed by $\mathrm{CO}$, before treating a more general numerical approach using a multi-ray quadrature of the stellar disk, a method applicable with any choice of limb darkening law.

\subsubsection{Case of a purely radially expanding flow}

If one assumes a uniformly bright stellar disk, the limb darkening law is given by

$\mathcal{D}\left(r, \mu^{\prime}\right)= \begin{cases}0, & -1 \leq \mu^{\prime} \leq \mu_{\star} \\ 1 / \pi, & \mu_{\star} \leq \mu^{\prime} \leq 1 .\end{cases}$
With the assumption that the velocity field is purely radial, the projected gradient of the projected velocity reduces to

$$
\begin{aligned}
(\boldsymbol{n} \cdot \nabla(\boldsymbol{n} \cdot \boldsymbol{v}))(\boldsymbol{r}) & =\mu^{\prime 2} \frac{\partial v_{r}}{\partial r}+\left(1-\mu^{\prime 2}\right) \frac{v_{r}}{r} \\
& =\left(\frac{1+\sigma \mu^{\prime 2}}{1+\sigma}\right)\left(\frac{\partial v_{r}}{\partial r}\right)
\end{aligned}
$$

where I have used the logarithmic derivative variable $\sigma \equiv$ $\frac{\partial \ln v_{r}}{\partial \ln r}-1$ in the last equality. Equation (1) can then be expressed in terms of the line acceleration for a point-star multiplied by the so-called finite disk correction factor $f$,

$\boldsymbol{g}_{\mathrm{rad}, \mathrm{CO}}^{\text {line }}(\boldsymbol{r})=f \frac{\sigma_{\mathrm{e}}^{1-\alpha} L_{\star}}{4 \pi c r^{2}} \frac{k}{\left(\rho v_{\mathrm{th}}\right)^{\alpha}}\left(\frac{\partial v_{r}}{\partial r}\right)^{\alpha}$

with

$f=\frac{(1+\sigma)^{1+\alpha}-\left(1+\sigma \mu_{\star}^{2}\right)^{1+\alpha}}{\sigma(1+\alpha)\left(1-\mu_{\star}^{2}\right)\left(1+\sigma^{\alpha}\right)}$

Note that here as well as below, I take what $\mathrm{CO}$ call the weak limit when I estimate the projected gradient of the projected velocity, i.e. its value is set to naught if negative, left as is if positive.

This method is advantageous because the radiative acceleration at $\boldsymbol{r}$ is obtained from the evaluation of a single expression based exclusively on local quantities.

\subsubsection{General case with a ray-quadrature of the visible stellar disk}

In this work, I want to investigate potential effects resulting from non-radial components of the line acceleration, which may be significant in the presence of localized surface brightness variations. In this case, one needs to evaluate numerically the integral in Eq. (1), by performing a ray quadrature of the visible stellar disk at $\boldsymbol{r}$.

In $\mathrm{CO}$, the spot influence (discussed in the next section of this paper) is computed under the assumption of a linear grey limb darkening. Thus, to avoid biases that could be introduced by adopting a different limb-darkening, I have made the same assumption. As explained in Cranmer \& Owocki (1995), this choice over that of a uniformly bright disk leads to a higher stellar mass loss rate by ca. $10 \%$ because for a given stellar luminosity, it enhances the radiative flux received in the radial direction at a given wind point. In the context of a surface bright spot, it will also tend to favor its influence along the local vertical.

The linear limb darkening law is given by,

$\mathcal{D}\left(r, \mu^{\prime}\right)=\left\{\begin{array}{lr}0, & -1 \leq \mu^{\prime} \leq \mu_{\star} \\ \left(2+3 \mu^{\prime \prime}\right) / 4 \pi, & \mu_{\star} \leq \mu^{\prime} \leq 1\end{array}\right.$

where $\mu^{\prime \prime} \equiv \cos \theta^{\prime \prime}$ is the angle cosine between the local vertical on the star $(\boldsymbol{r})$ and the direction to the wind point $(\boldsymbol{n})$. It is related to $\mu^{\prime}$ through

$\mu^{\prime \prime}=\sqrt{\frac{\mu^{\prime 2}-\mu_{\star}^{2}}{1-\mu_{\star}^{2}}}$ 
Hence, the general line acceleration expression becomes

$$
\begin{aligned}
\boldsymbol{g}_{\mathrm{rad}}^{\text {line }}(\boldsymbol{r})= & \frac{\sigma_{\mathrm{e}}^{1-\alpha} k L_{\star}}{64 \pi^{2} R_{\star}^{2} c\left(\rho v_{\mathrm{th}}\right)^{\alpha}} \\
& \times \int_{0}^{2 \pi} \mathrm{d} \phi^{\prime} \int_{\mu_{\star}}^{1} \mathrm{~d} \mu^{\prime}\left(2+3 \sqrt{\frac{\mu^{\prime 2}-\mu_{\star}^{2}}{1-\mu_{\star}^{2}}}\right) \\
& \times(\boldsymbol{n} \cdot \nabla(\boldsymbol{n} \cdot \boldsymbol{v}))^{\alpha} \boldsymbol{n} .
\end{aligned}
$$

To remain general, I do not assume any symmetry in the velocity field or base brightness properties. In this case, the projected gradient of the projected velocity $(\boldsymbol{n} \cdot \nabla(\boldsymbol{n} \cdot \boldsymbol{v}))$ retains its dependence on all three variables $\left(r, \theta^{\prime}, \phi^{\prime}\right)$, leading a priori to non-zero terms for each of the three projected components of the radiative acceleration along $\boldsymbol{n}_{r}, \boldsymbol{n}_{\theta}$ and $\boldsymbol{n}_{\phi}$. Following previous works (Batchelor 1967; Koninx 1992; Cranmer \& Owocki 1995),

$$
\begin{aligned}
(\boldsymbol{n} \cdot \nabla(\boldsymbol{n} \cdot \boldsymbol{v}))(\boldsymbol{r})= & \left(\frac{\partial v_{r}}{\partial r}\right) a_{1}^{2}+\left(\frac{1}{r} \frac{\partial v_{\theta}}{\partial \theta}+\frac{v_{r}}{r}\right) a_{2}^{2} \\
& +\left(\frac{1}{r \sin \theta} \frac{\partial v_{\phi}}{\partial \phi}+\frac{v_{r}}{r}+\frac{v_{\theta}}{r} \cot \theta\right) a_{3}^{2} \\
& +\left(\frac{\partial v_{\theta}}{\partial r}-\frac{v_{\theta}}{r}+\frac{1}{r} \frac{\partial v_{r}}{\partial \theta}\right) a_{1} a_{2} \\
& +\left(\frac{\partial v_{\phi}}{\partial r}+\frac{1}{r \sin \theta} \frac{\partial v_{r}}{\partial \phi}-\frac{v_{\phi}}{r}\right) a_{1} a_{3} \\
& +\left(\frac{1}{r} \frac{\partial v_{\phi}}{\partial \theta}+\frac{1}{r \sin \theta} \frac{\partial v_{\theta}}{\partial \phi}-\frac{v_{\phi}}{r} \cot \theta\right) a_{2} a_{3}
\end{aligned}
$$

where

$a_{1}=\mu^{\prime}$

$a_{2}=\sqrt{1-\mu^{\prime 2}} \cos \phi^{\prime}$

$a_{3}=\sqrt{1-\mu^{\prime 2}} \sin \phi^{\prime}$.

I select the rays using a Gauss-Legendre quadrature for both the $\mu^{\prime}$ and $\phi^{\prime}$ integrations. The actual number of rays used depends on the angular resolution of the simulation, the scale of variations imposed at the base and the limits imposed on the computational time. As explained below, I used quite large large-scale brightness variations so that a ray quadrature with $\left(n_{\mu^{\prime}}, n_{\phi^{\prime}}\right)=(4,12)$ was fine enough to capture the complexity of the surface pattern.

\subsection{Wind properties obtained with the multi-ray radiative acceleration}

Before discussing the properties of the perturbed force, it might be worthwhile to describe few characteristics of the unperturbed outflow subject to this multi-ray-computed radiation force, which provides the three components of the radiative acceleration rather than the radial one alone. Without surface perturbations, the azimuthal symmetry is preserved and thus, I present here some results obtained in $2 \mathrm{D}$ simulations, covering from the stellar surface to $30 R_{\star}$ and from the rotation axis down to the equator, using stellar and numerical parameters described later in Sect. 3. These results are discussed here

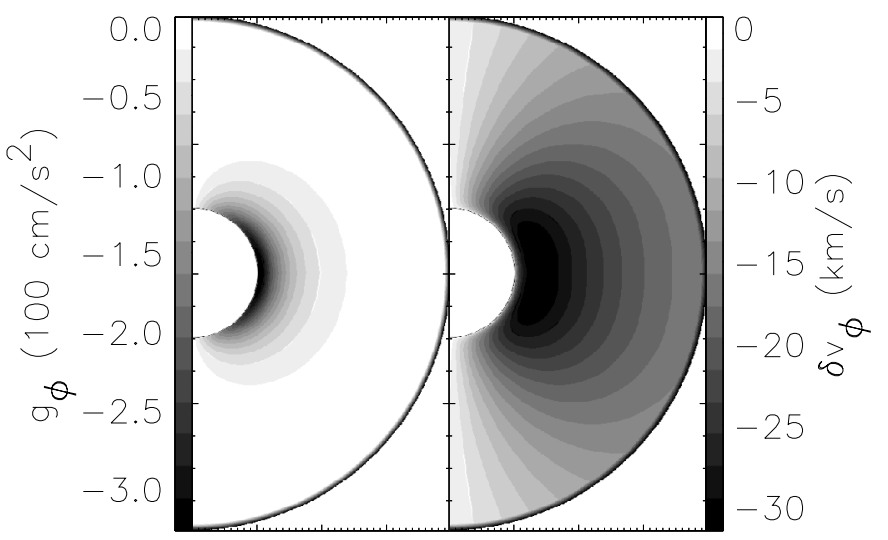

Fig. 1. Left: gray-scale image of the azimuthal radiative acceleration resulting from the fore-aft asymmetry in the velocity gradient along prograde-retrograde rays. I only show the inner wind (up to $4 R_{\star}$ ), i.e. the region where this spin-down effect has a noticeable impact on the wind dynamics. Right: gray-scale image of the resulting azimuthal spin down of wind rotation, shown here as the deviation of the relaxed azimuthal velocity compared to its value obtained under the neglect of non-radial components of the radiative acceleration.

nonetheless because they represent very general properties of rotating line-driven winds.

As discussed in Gayley \& Owocki (2000), stellar rotation introduces an asymmetry in the line-of-sight velocity gradient along prograde and retrograde rays as seen from any wind point. In particular, when the radial variation of the azimuthal velocity falls below the rigid-body form $v(r, \theta, \phi)=\Omega r \sin \theta$, the azimuthal line-force is negative and leads to a partial spindown of the wind. I reproduce this effect here and obtain a wind spin-down of ca. $30 \%$ compared to the situation where only the radial radiation force is included (Fig. 1). This is a nice check on the code, giving a wind spin-down magnitude comparable to that found in Gayley \& Owocki (2000) for a fast rotating B star.

Stellar rotation has also indirect influences on the wind dynamics. The centrifugal acceleration at the stellar surface is maximum in the equatorial plane and decreases towards the pole. This introduces a modulation of the effective gravity with latitude. In simulations for a star like $\zeta$ Puppis (Sect. 3), which rotates only moderately fast $\left(v_{\text {rot }}=230 \mathrm{~km} \mathrm{~s}^{-1}\right.$, Sect. 3$)$, this results in a higher terminal speed for higher latitudes (of about $15 \%$ ). Consequently, from the star surface, the velocity gradient along lines of sight tilted towards the pole will be higher than in other directions, thus making the radiative acceleration stronger in the pole-ward direction. This property acts against the tendency for material located at high latitudes to drift towards the equatorial plane, under the constraint of angular momentum conservation (Bjorkman \& Cassinelli 1993; Petrenz 1999; Petrenz \& Puls 2000). In simulations, I find that the maximum latitudinal velocity occurs for regions located around a latitude of $\sim 45 \mathrm{deg}$, but has only a magnitude of ca. $15 \mathrm{~km} \mathrm{~s}^{-1}$, i.e. not even reaching the adopted isothermal sound speed. Note that this equatorward-drift would be strengthened if the rotational velocity of the star was larger. For example, in Be star models, for which the rotational velocity is comparable to the terminal velocity of the flow, this drift is indeed predicted 
to be maximum, leading, in numerical simulations, to the formation of a Wind Compressed Disk (Bjorkman \& Cassinelli 1993; Owocki et al. 1994; see Owocki et al. 1996 for key physical counter-arguments). But here, for the modest rotational velocity considered, latitudinal motions will play only a little role in controlling the overall wind dynamics.

In the current form of the code, the stellar oblateness and its possible association with gravity darkening are not included. As discussed by Cranmer \& Owocki (1995) and Owocki et al. (1996), these two issues can greatly affect the morphology of a line-driven wind, favoring stellar mass loss in the polar region of the fastest rotating hot stars. Because I neglect gravity darkening, I tend to overestimate the flux contribution of equatorial regions. But because I also neglect oblateness, I underestimate the pole-ward tilt of the radiative acceleration. Thus, it seems that these two simplifications play against each other and might in fact cancel. Of course, I intend in the future to extend the present formalism of my code to treat explicitly these two effects.

\subsection{Radiative acceleration enhancement}

The general perspective of $\mathrm{CO}$ was to investigate the impact on a line-driven wind of variations based at the stellar surface. The large amplitude and long term variability identified in UV P-Cygni and optical emission lines of $\mathrm{O}$ and Wolf-Rayet stars suggest variations in wind density and/or velocity requiring an external perturbation. The details of the cause of such variations is not the center of the present problem; rather I assume such variations exist and investigate their impact on a line-driven wind (see $\mathrm{CO}$ for a discussion on this issue).

$\mathrm{CO}$ modeled such variations as localized Gaussian spots and investigated the induced perturbation on the radial component of the radiation force, while neglecting any lateral effect. I give a synopsis of their treatment in Sect. 2.4.1, which permits a quick evaluation of the radiation force at any wind point. Using the same spot properties, I discuss in Sect. 2.4.2 a more general numerical method that computes the three components of the perturbed radiation force, for any latitude of the surface spot.

\subsubsection{The approach of $\mathrm{CO}$}

In the $2 \mathrm{D}$ simulations of $\mathrm{CO}$, the perturbed radiative acceleration is equal to its unperturbed value scaled by a geometrical factor $\xi_{\mathrm{CO}}(r, \phi)$ function of the height above the photosphere and the angular distance between the wind point and the spot, as seen from the star centre. The azimuthal modulation is taken Gaussian (CO) so that,

$\xi_{\mathrm{CO}}(r, \phi)=1+A \gamma(r) \exp \left[-\left(\phi-\phi_{\text {spot }}\right)^{2} / \sigma^{2}\right]$

$A$ is the amplitude of the spot (positive for bright spots and negative for dark ones), $\gamma(r)$ the radial-modulation scale factor, and $\phi_{\text {spot }}$ the azimuthal angle of the spot, corotating with the stellar surface. The full width at half maximum of the spot is given by $\Phi \equiv 2 \sigma \sqrt{\ln 2}$. CO use an analytical expression for the radial-modulation of the force enhancement, quoted here for completeness,

$\gamma(r)=\frac{1}{2}\left[1+\left(\frac{1-\mu_{0}^{2}}{1-\mu_{\star}^{2}}\right)-\left(\frac{\mu_{0}^{2}-\mu_{\star}^{2}}{1-\mu_{\star}^{2}}\right)^{3 / 2}\right]$

where $\mu_{0}$ represents the visible edge of the spot as seen from $\boldsymbol{r}$, i.e.

$\mu_{0}= \begin{cases}\mu_{\star}, & r \cos (\Phi / 2) \leq R_{\star} \\ \sqrt{1-R_{\star}^{2} \sin ^{2}(\Phi / 2) / S^{2}}, & r \cos (\Phi / 2)>R_{\star}\end{cases}$

and $S$ is the distance between the field point and the edge of the spot, i.e. $\sqrt{r^{2}+R_{\star}^{2}-2 r R_{\star} \cos (\Phi / 2)}$.

Hence, one sees that the perturbed force is quickly obtained by applying a geometrical scaling to the unperturbed value. This geometrical factor can be evaluated at the start of the simulation so that the treatment of brightness variations does not increase directly its computational cost.

\subsubsection{General case. Force morphology: $g_{r}, g_{\theta}, g_{\phi}$}

In general 3D investigations, one needs to supply not just one but two angles to track the position of the spot corotating with the stellar surface, i.e. $\theta_{\text {spot }}$ and $\phi_{\text {spot }}$. Since I impose no latitudinal base motions, the angle $\theta_{\text {spot }}$ remains fixed in time. Since I do not consider any differential angular rotation between distinct latitudes, the angle $\phi_{\text {spot }}$ will be incremented at the same rate at all latitudes, so that for two consecutive times $t_{0}$ and $t$, one has

$\phi_{\text {spot }}(t)=\phi_{\text {spot }}\left(t_{0}\right)+\Omega\left(t-t_{0}\right)$.

As in $\mathrm{CO}$, the angular variation of the force enhancement is assumed to be Gaussian about the spot direction $\boldsymbol{n}_{\text {spot }}$, described by the angles $\theta_{\text {spot }}$ and $\phi_{\text {spot }}$. Hence, the spot influence (discarding dynamical considerations for now) is identical in all directions making the same angle with the direction $\boldsymbol{n}_{\text {spot }}$.

The radiative acceleration at $\boldsymbol{r}$ is computed by scaling, with the desired choice of spot amplitude $A$, the integrand in Eq. (9) by the factor,

$\xi\left(\boldsymbol{n}_{0}, \boldsymbol{n}_{\text {spot }}\right)=1+A \exp \left[-\left(\frac{\arccos \left(\boldsymbol{n}_{0} \cdot \boldsymbol{n}_{\text {spot }}\right)}{\sigma}\right)^{2}\right]$

where $\boldsymbol{n}_{0}$ is the unit vector defined by the spherical polar angles $\left(\theta_{0}, \phi_{0}\right)$ referring to the intersection point between a given wind-centred ray $\left(\mu^{\prime}, \phi^{\prime}\right)$ and the stellar surface. This factor directly scales the projected velocity gradient along the direction $\left(\mu^{\prime}, \phi^{\prime}\right)$, hence coupling explicitly surface brightness variations and local dynamical properties (this is one of the differences with CO whose approach decouples the local dynamics and the geometrical scaling resulting from brightness variations).

In practice, at the start of the simulation, I determine for all wind heights and latitudes the set of angles $\left(\theta_{0}, \phi_{0}\right)$ for a meridional slice at $\phi=0$. This overall task is trivial since I do not include the oblateness of the star: from a wind location and the set $\left(\theta^{\prime}, \phi^{\prime}\right)$, I determine the Cartesian coordinates of the stellar surface point in the wind-centred frame, then in the starcentred frame, and finally obtain the spherical angles $\left(\theta_{0}, \phi_{0}\right)$. 

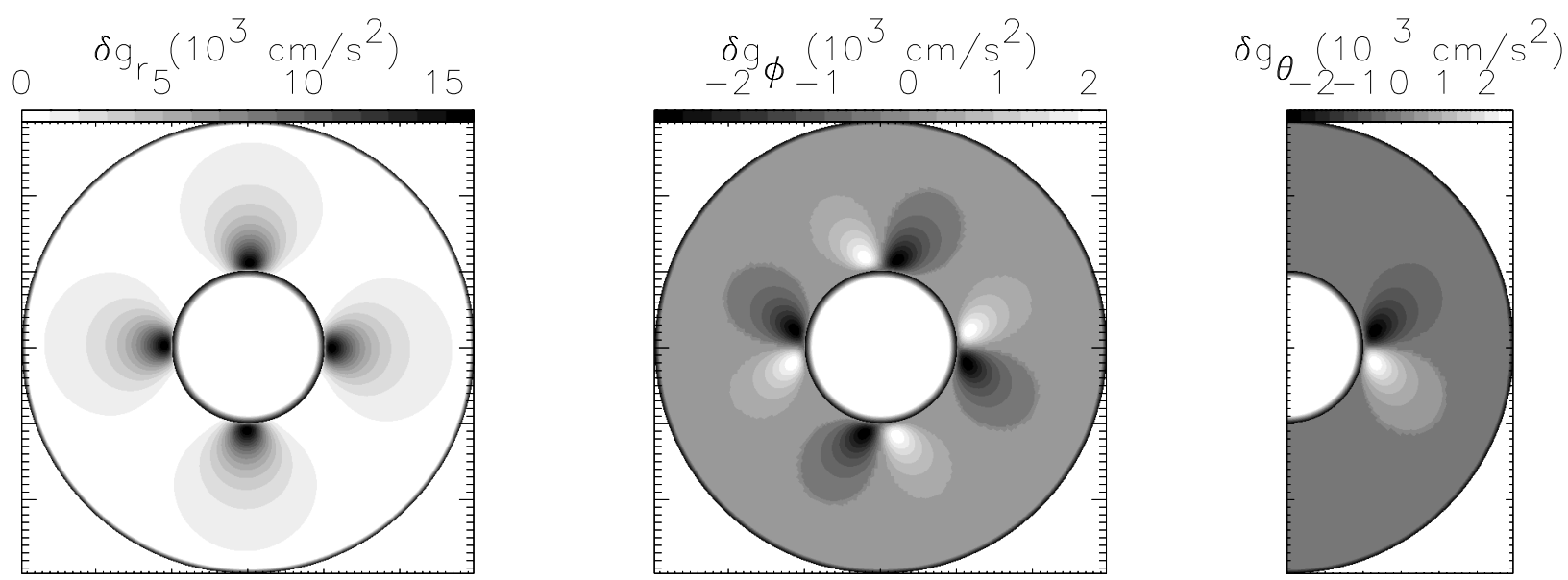

Fig. 2. Gray-scale cross sections of the deviation of the perturbed radiative acceleration from its unperturbed value, showing the radial, latitudinal and azimuthal components for a model with 4 equally spaced bright spots $(A=2)$ over 360 deg in the equatorial plane. The perturbed radiative acceleration deviates non-negligibly from its unperturbed form only in the inner wind, so I limit the radial extent in the figure to $3 R_{\star}$. $\delta g_{r}$ and $\delta g_{\phi}$ are shown in the equatorial plane. $\delta g_{\theta}$ is shown in a meridional slice at the spot azimuthal position. The latitudinal radiative acceleration in the equatorial plane is zero, hence not shown. Similarly, the azimuthal perturbed radiative acceleration in the chosen meridional slice is zero. I find that the latitudinal and azimuthal perturbed components have similar properties, in agreement with the assumption of a Gaussian spot whose influence is solely a function of the angular distance between the point of interest and that of the spot. The general properties shown here for equatorial spots are also found for off-equatorial ones. The magnitude of the lateral components of the radiative acceleration is of the order of the effective gravity at the stellar surface, i.e. $g_{\mathrm{eff}}=\mathrm{GM} / R_{\star}^{2}(1-\Gamma) \sim 2970 \mathrm{~cm} \mathrm{~s}^{-2}$, where $\Gamma$ is the Eddington factor (see Sect. 3 for the choice of stellar parameters).

For different azimuths, I apply a translation in the direction $\phi$ to know the adequate value of $\phi_{0}$ for the given ray.

I compute the multi-ray radiation force with a set of $4 \times$ 12 rays for the $\left(\mu^{\prime}, \phi^{\prime}\right)$ stellar disk quadrature, which requires that the angular scale of surface variations be quite large. I find that the impact of spots characterized by a full width half maximum of $20 \mathrm{deg}$ is well modeled with such choice of ray quadrature. I have not used smaller scale spots since this makes the numerical computation a lot more lengthy while not modifying the conceptual aspects drawn with larger ones.

In Fig. 2, I show a gray-scale representation of the deviation of the three components of the perturbed radiative acceleration from their unperturbed value, $\delta \boldsymbol{g}_{\mathrm{rad}}^{\text {line }}$, for the situation where 4 bright spots have been placed in the equatorial plane, at intervals of $90 \mathrm{deg}$. At the vertical of a spot, for higher wind heights, the spot represents a smaller fraction of the visible stellar disk so that at larger distances, all three components of $\delta \boldsymbol{g}_{\mathrm{rad}}^{\text {line }}$ drop to zero. Hence, Fig. 2 only shows the first three stellar radii where $\delta \boldsymbol{g}_{\text {rad }}^{\text {line }}$ deviates significantly from zero. From left to right, I show the radial and azimuthal components in the equatorial plane, and the latitudinal component through a meridional slice cutting through the center of one of the spots.

The perturbed radial radiative acceleration $\delta g_{r}$ has similar properties to those derived by $\mathrm{CO}$ and shown in their Fig. 1. I also find that its latitudinal variation (not shown here) follows the azimuthal pattern shown in the present Fig. 2, i.e. the spot influence in the radial direction is to a first order symmetric about the spot position (in reality, the presence of the rotational differential velocity introduces a minor asymmetry in this acceleration between the prograde and retrograde directions).

The latitudinal and azimuthal patterns for the perturbed radiation force are similar (of the order of the surface effective gravity for $A=2$ ), with the differences arising from the presence of an azimuthal velocity and its latitudinal variation. A bright equatorial spot enhances the radiative acceleration along directions that intersect it: it spins up the rotating wind ahead of it, and spins down the rotating material lagging behind. This behavior contains an interesting implication. The induced spinup from such a bright spot could perhaps impart material with enough angular momentum to stay in orbit, hence playing a role in the making of circumstellar disks (Owocki 2002).

For the same reason, the perturbed latitudinal radiative acceleration has the tendency to deflect the material away from the spot latitude. Together, the lateral components of the radiative acceleration have the ability to modify the dynamics of the wind just above a surface spot, reducing the angular confinement over which the spot influences wind driving. In order to favor the dynamical influence of spots in the lateral direction, all simulations in Sect. 3 are performed with $A=2$, while in reality, it is not clear how strong such surface perturbations might be.

\section{Numerical results}

As in CO, I choose the model parameters to suit the stellar and wind properties of the O4f supergiant star $\zeta$ Puppis, i.e. I take $M_{\star}=60 M_{\odot}, R_{\star}=19 R_{\odot}, L_{\star}=8 \times 10^{5} L_{\odot}$ and $T_{\star}=42 \mathrm{kK}$. I set the rotational velocity to $230 \mathrm{~km} \mathrm{~s}^{-1}$, the measured $V_{\mathrm{eq}} \sin i$ for $\zeta$ Puppis. I also neglect the small $(\sim 7 \%)$ oblateness of the star. The wind is also assumed to be isothermal, the temperature being fixed at $T_{\star}$, corresponding to a uniform sound speed of $24 \mathrm{~km} \mathrm{~s}^{-1}$. The line-driving constants are $\alpha=0.6$ and $k=0.15$.

The primary concern in this work is to delineate the consequences of including non-radial components of the radiation 

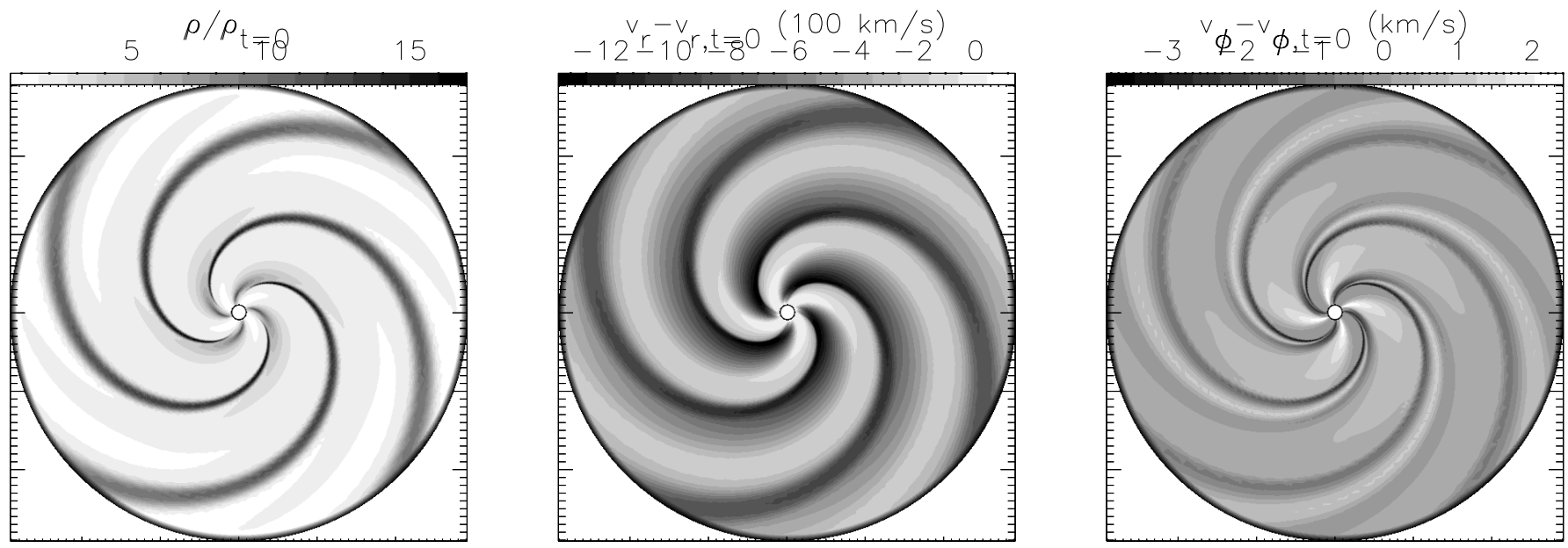

Fig. 3. The CO approach. Gray-scale image of the deviation of the relaxed velocity field and density from their initial values using the form of the perturbed radiative acceleration of $\mathrm{CO}$ and $A=2$. The initial conditions of the simulation are the relaxed wind properties obtained by running the simulation under identical model parameters but nulling the spot amplitude. The numerical domain is limited to $2 \mathrm{D}$, as in $\mathrm{CO}$, and covers a quadrant of the equatorial plane of the rotating star. The latitudinal velocity is naught, hence not shown here. The radial grid extends out to $30 R_{\star}$

force, as well as addressing the limitations associated with restricting the simulation domain to 2D. Hence, I use a fixed set of spot properties, i.e. $A=2, \Phi=20 \mathrm{deg}$. Under such an assumption, I study the CIR properties computed using the purely radial $(\mathrm{CO})$ and the multi-ray radiation force in $2 \mathrm{D}$ (Sect. 3.1), the multi-ray radiation force in $3 \mathrm{D}$ for spots in the equatorial plane (Sect. 3.2) and at a non-zero latitude (Sect. 3.3).

The numerical procedure I follow in my simulations is to start by generating fluid properties for the unperturbed case. For 2D simulations, I start by running a $1 \mathrm{D}$ simulation in the equatorial plane, and then re-map it onto a $2 \mathrm{D}$ equatorial wedge. I then re-run the code using the two choices for the radiation force, i.e. the $\mathrm{CO}$ or multi-ray approach. For 3D simulations, this requires running the code for a meridional slice, using azimuthal symmetry. For such a relaxed 2D meridional slice, I then map the generated velocity vector and density onto a 3D octant of a sphere, assign spot properties and restart the computation using the same multi-ray radiation force computation. Finally, to ensure that the wind has relaxed fully to the model contraints, all simulations are run for $400000 \mathrm{~s}$, which well exceeds, at all latitudes, the wind crossing time out to 30 stellar radii.

\subsection{Equatorial bright spot: Comparison between equatorial properties for $C O$, and multi-ray $2 D$ and $3 D$ simulations}

In Figs. 3-5, I show gray-scale images of the deviation of fluid properties from their initial unperturbed relaxed values (obtained with identical model parameters). Figure 3 shows the perturbed density, radial and azimuthal velocities for the $\mathrm{CO}$ case, i.e. under the assumption that the perturbations to linedriving brought about by the surface spots are only reflected in its azimuthally modulated radial component, while in Fig. 4, I show the properties obtained when the perturbation to all three components of the radiative acceleration are considered.
In Fig. 5, I show the wind properties obtained under the same assumptions as for Fig. 4, but extending the simulations domain from $2 \mathrm{D}$ to $3 \mathrm{D}$. Hence, I wish to discuss model results along two directions. First, I address the importance of lateral effects in 2D equatorial plane simulations by comparing the $\mathrm{CO}$ and "multi-ray" cases. Second, I address the 3D effects by comparing the "multi-ray" case in 2D and 3D runs.

\subsubsection{Dynamical impact of the perturbed lateral radiative acceleration}

Inspection of Figs. 3 and 4 reveals that the CIR pattern is similar, as seen in the perturbed density and velocity fields, although the CIR pattern is much sharper in Fig. 3. Although the spot characteristics are identical in the two simulations, the magnitude of the resulting perturbations on the wind is indeed different.

The origin of this difference comes from accounting for the perturbed azimuthal radiative acceleration. It introduces a significant spin-up of the wind ahead of the spot, and a further spin-down of the wind in the back of the spot. Near the stellar surface, as shown in Fig. 2, the perturbed azimuthal and radial radiative accelerations are of the same order and therefore the former can play a dynamical role. Eventually, this leads to extrema for the perturbed azimuthal velocity field about 50 times as large in the multi-ray approach compared to the CO case. The indirect effect is to azimuthally broaden as well as reduce the magnitude of the spot influence, which can be seen in the resulting CIR pattern. In the $\mathrm{CO}$ case, the maximum of the density contrast $\max \left(\rho / \rho_{0}\right)$ is about twice as large, and similarly the velocity contrast $\max \left|v_{r}-v_{r_{0}}\right|$ is $50 \%$ larger compared to the "multi-ray" approach.

Note however that without definite knowledge of the magnitude of surface brightness perturbations, these two alternative wind properties cannot be distinguished easily. The modification of the CIR pattern is only modest, and the magnitude of 

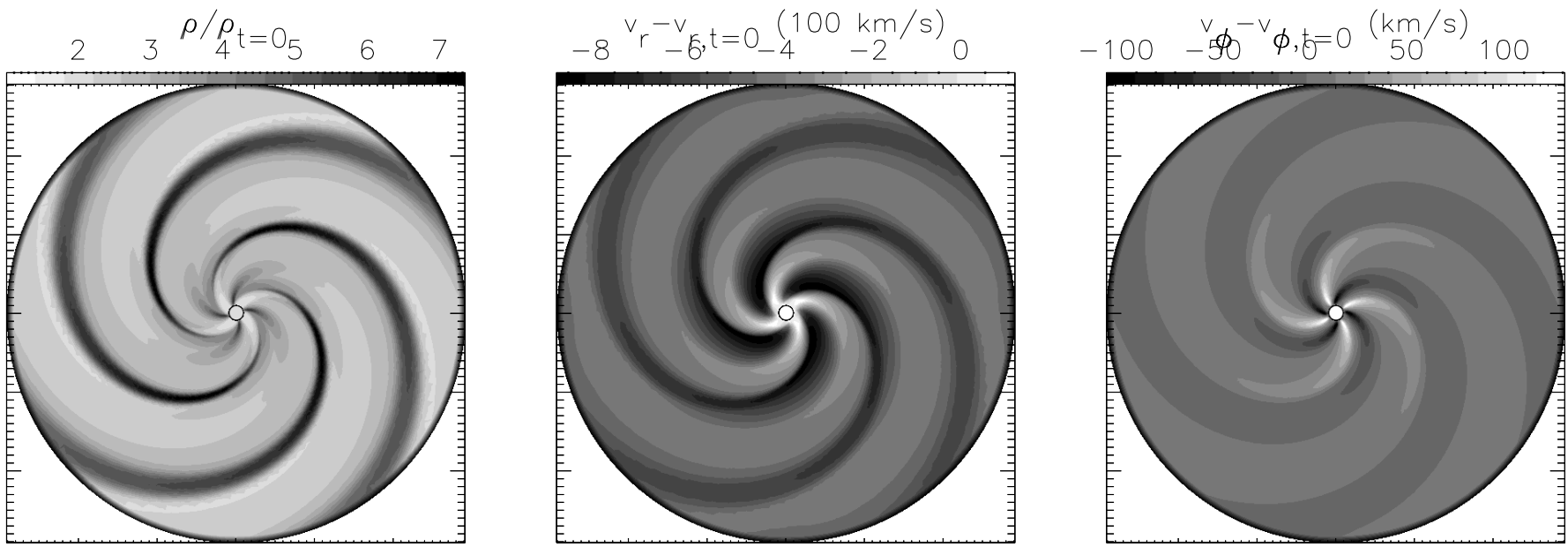

Fig. 4. The multi-ray approach. Same as Fig. 2 but using the multi-ray computation of the radiative acceleration. This permits in particular to account for the dynamical influence of its lateral component, the associated wind spin-down and its modulation due to the presence of the surface bright spot. Notice that the qualitative properties of CIRs are essentially the same as those obtained using the approach of CO. But quantitatively, differences are present: the overall CIR density compression and velocity kink have their magnitude reduced by 100 and $50 \%$ respectively. The azimuthal velocity field is more strikingly affected by the presence of the spots, the wind material showing a strong spin-up ahead of the spot, and a spin-down of similar magnitude in the trail of the spot (superimposed on the wind spin-down effect, Fig. 1, Sect. 2.3). The contrast with the unperturbed value is about 50 times greater than obtained in $\mathrm{CO}$ (Fig. 3). The dynamical influence of the surface spots is also more spread in azimuth, so that only very restricted wind regions remain unperturbed. Here again, the radial grid extends out to $30 R_{\star}$.
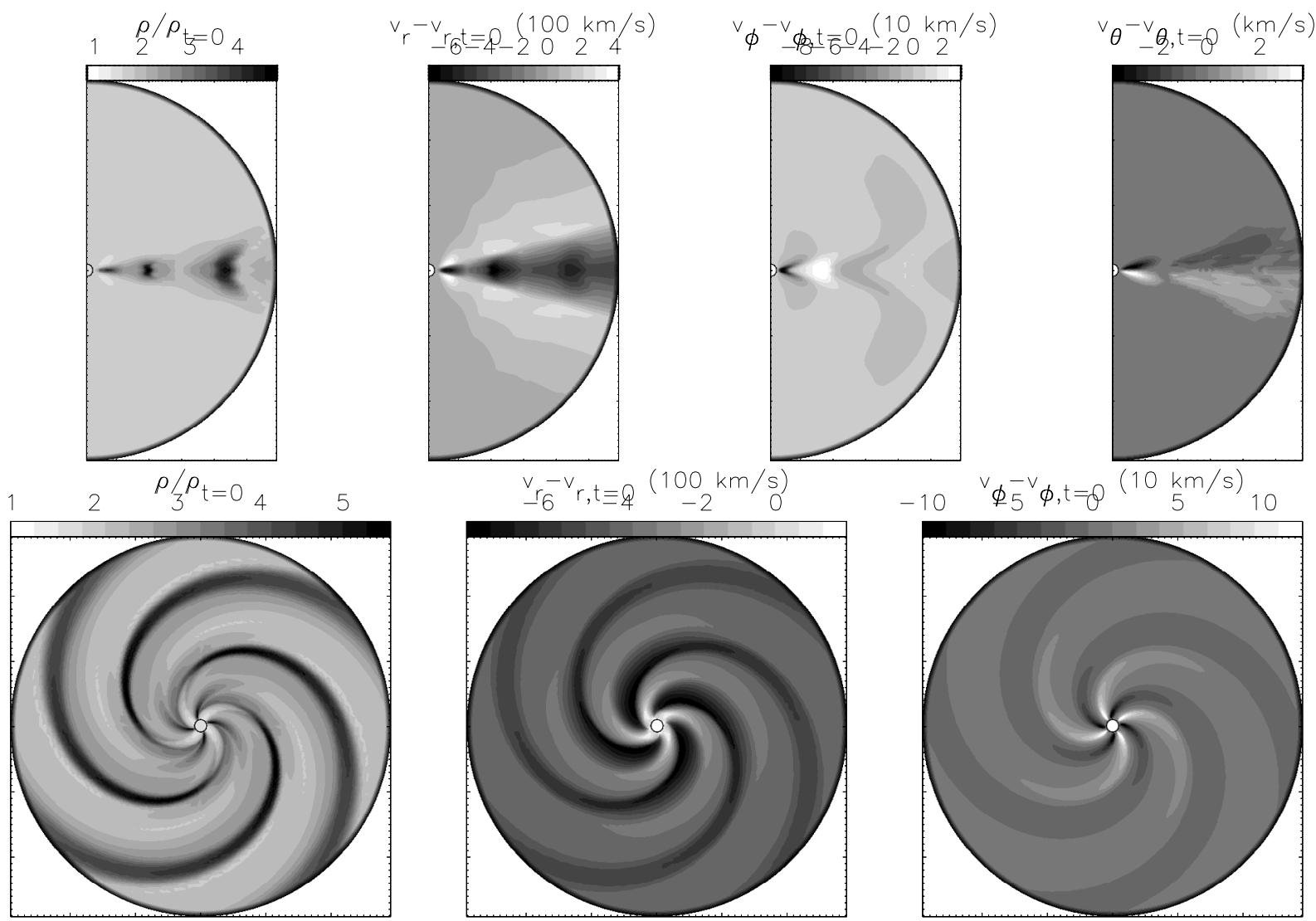

Fig. 5. Set of gray-scale images of the deviations of the fluid variables compared to initial relaxed values obtained under identical conditions but $A=0$. The four spots are placed in the equatorial plane of the rotating star. The latitudinal velocity is not shown in the equatorial plane since it is zero there. Again, the CIR properties are consistent with what was obtained in 2D, with the CO and multi-ray approaches. But the inclusion of the third dimension (latitude) leads to a further reduction in the magnitude of the density contrast and the velocity kink associated with the CIRs. Also, notice how the CIR pattern remains confined to the equatorial plane, with a latitudinal extent of the order of the full-width-half-maximum adopted for the surface spots. The radial grid extends out to $30 R_{\star}$. 
the perturbed lateral velocity field is comparable to that corresponding to micro-turbulent motions in hot stars (Hubeny et al. 1991).

\subsubsection{D effects}

Having found that the dynamical effects of the perturbed lateral radiative acceleration on the fundamental CIR properties discussed by $\mathrm{CO}$ are modest, I wish now to discuss the effects associated to the restriction of the simulation domain to $2 \mathrm{D}$. Thus, I have performed 2D and 3D simulations with the multiray computation of the radiation force and for spots at zero latitude (for a proper comparison since the method of $\mathrm{CO}$ only applies directly to $2 \mathrm{D}$ simulations in the equatorial plane). The relevant figures to compare are now Figs. 4 and 5.

The radially expanding outflow can now expand into both azimuthal and latitudinal directions. This extra-dimension allows compressed material to move into one more dimension, and as expected, this leads to weaker compressions. Indeed, the maximum of the density contrast in the 3D simulation is $15 \%$ less than in the equivalent $2 \mathrm{D}$ simulation, with a similar decrease for $\max \left|v_{r}-v_{r_{0}}\right|$. However, the perturbed lateral velocity field is essentially unchanged. So, I can conclude that the $3 \mathrm{D}$ effects on the CIR properties are again modest, i.e. the $2 \mathrm{D}$ description captures their key properties.

An additional feature of 3D simulations is the ability to determine the latitudinal extent over which the wind is effectively perturbed by the spot. In their spectral synthesis computations, $\mathrm{CO}$ assumed that this value was given by the full width at half maximum of the Gaussian spot. In the top panels of Fig. 5, I show meridional cuts through the simulation domain (only the northern hemisphere is calculated for, the density and velocity fields in the southern hemisphere are given by the adopted symmetry for the problem, see Sect. 2). The angular extent over which the perturbation dominates is indeed confined within a latitudinal angle of ca. $20 \mathrm{deg}$, validating the assumption of CO. This result is tied to the fact that the latitudinal velocity field is vanishingly small in the equatorial regions for both perturbed and unperturbed components so that most of the CIR pattern is characterized by the angular extent over which the radial radiative acceleration is modified by the spot.

In the next section, I discuss the CIR properties obtained when the surface brightness variations are positioned at offequatorial latitudes.

\subsection{Off-equatorial bright spot}

The boundary implementation and the multi-ray radiation force computation are flexible so that no modification to the code needs to be made when surface spots are moved away from the equatorial region. However, I keep the spots away from the rotation axis since the periodicity in the azimuthal direction can lead to an overlap of the influence of two successive spots, which would then result in brightening the polar regions while I wish to impose azimuthally confined brightness variations (hence not covering the full $360 \mathrm{deg} \phi$ range).
In Fig. 6, I show the perturbed fluid properties (same display convention as for Fig. 5) arising from the presence of bright spots lying at a 45 deg latitude. The bottom layer does not represent the equatorial plane of the star but corresponds to the conic shell revolving around the rotating axis and lying at the spot latitude. Hence, here, the opening angle of this conic shell is $45 \mathrm{deg}$ while for the display of the equatorial plane properties (bottom row of Fig. 4), such a conic shell would have an opening angle of $90 \mathrm{deg}$. Because of the reflecting boundary condition of the equatorial plane, and the $90 \mathrm{deg}$ periodicity in azimuth, I now have a total of 8 spots, rather than 4 for the choice of equatorial spots.

A key finding is that the latitudinal extent over which the wind is perturbed is independent of the spot latitude. This is visible from the meridional slice of the density contrast, which is similar to its counterpart in Fig. 5. Again, because the latitudinal motions are negligible, the perturbed wind is confined to a conic shell whose thickness is of the order of the full-widthhalf-maximum of the spot. This conic shell layout is shown in the bottom row of Fig. 6. Hence, the spiraling pattern advects at a constant latitude, stretched further in radius as one inspects retrograde azimuths, but staying at the same angular distance from the rotation axis and the reflecting plane. This occurs for the same reason that was discussed in Sect. 2.4 and that explained the essentially radial stretching of wind streamlines from the balance between the slight pole-ward tilt of the radiative acceleration and the equator-ward motion of wind particles to preserve angular momentum. Again, this property could be somewhat altered for stars close to critical rotation, where the combined effects of oblateness and gravity darkening would tend to tilt the material towards the rotation axis.

The perturbed azimuthal velocity contrast is unaffected by the spot latitude, but its radial component and the density contrast have bigger extrema than when the spots are at zero latitude. This results from the crowding of spots in the polar region so that as material advects away from the surface, it starts seeing nearby spots invisible at the base. Hence, the mass loss is not affected by this overall polar brightening, but instead as wind particles advect further out, they get an "after-burn" effect when they start seeing nearby spots that were below their "horizon" at lower heights. Quantitatively, at colatitudes inferior to that of the spot, the over-all wind has a $25 \%$ larger terminal velocity than the unperturbed wind, whatever the spot azimuthal positions. This spot crowding is akin the polar brightening mentioned above.

Finally, I present in Fig. 7 a 3D view of the CIRs presented so far by means of 2D cuts in Figs. 5 and 6 . These figures are generated using a ray-tracing technique (Ruffert 1991) based on basic radiative transfer concepts. The observer is placed 1200 stellar radii away, at an angle of 30 (left panel) and $20 \mathrm{deg}$ (right panel) above the equatorial plane $x y$ of the system. Also for clarity, a color-coding is applied to visualise the projected position of the wind material along the $y$-axis, perpendicular to the page. To further simplify the interpretation, the observer is rotated around the $z$-axis by an angle of $30 \mathrm{deg}$. One can thus distinguish between the front and the back of the star: blue (red) color is used for material whose spectroscopic imprint in e.g. P-Cygni profiles would appear blue-shifted 


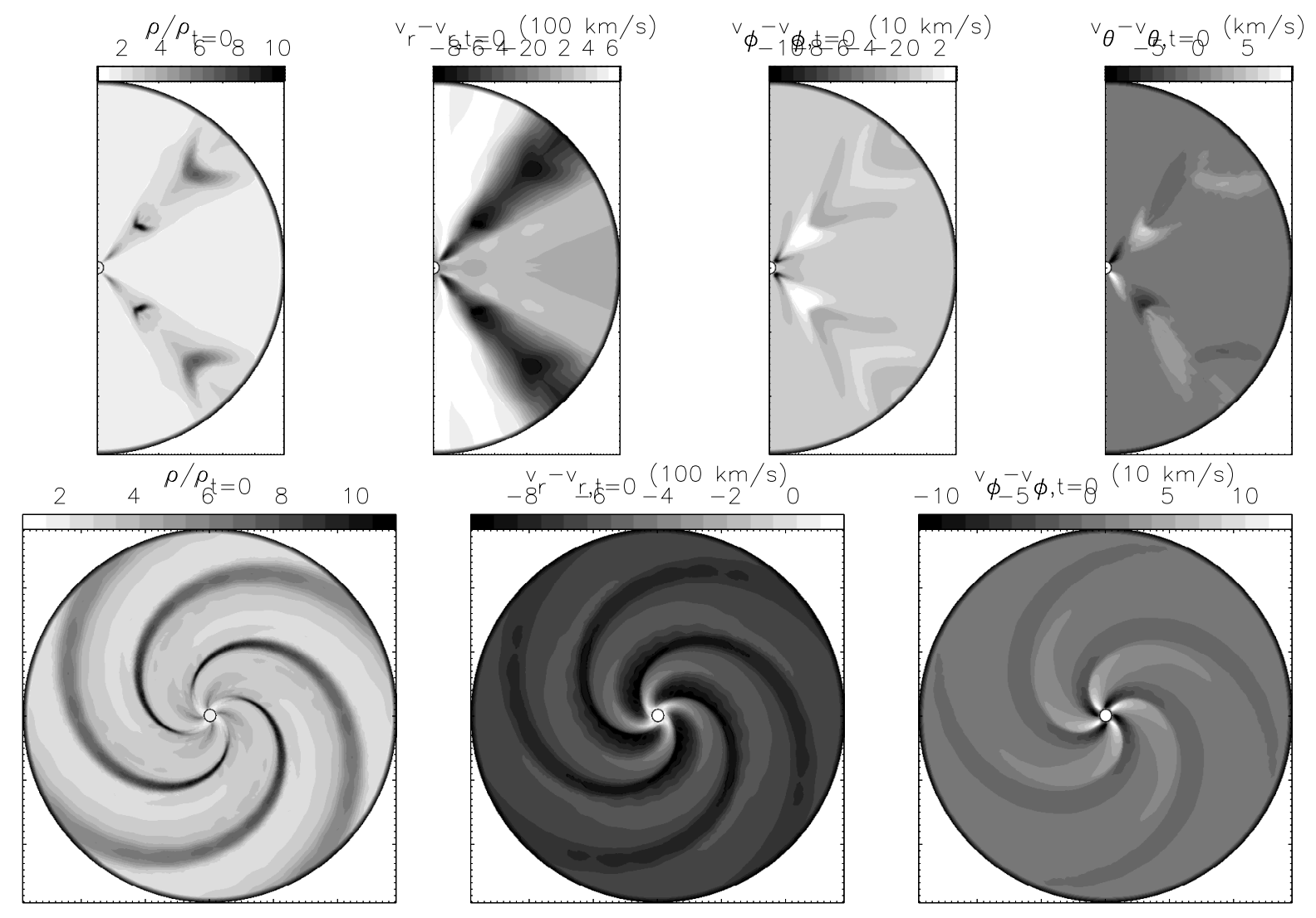

Fig. 6. Set of gray-scale images of the deviations of the fluid variables compared to initial relaxed values obtained under identical conditions but $A=0$. The four spots (per hemisphere) are placed at latitudes $\pm \pi / 4$. The bottom row shows a $2 \pi$ cut at the spot latitude, which can be viewed as a conic shell revolving around the rotating axis. This is shown to highlight the similarity of the radial-azimuthal properties of corotating interaction regions, irrespective of the latitude of the surface spots perturbing the outflow. Together with the vanishingly small latitudinal velocity field, this demonstrates that the CIR pattern advects at constant latitude, being confined to a conic shell centered on the spot latitude and having a latitudinal extent of the order of the full-width-half-maximum of the surface spots. The radial grid extends out to $30 R_{\star}$

(red-shifted) from line center (see Fig. 7 for more details and the electronic version of this article for a color version of this figure). Note that this color-coding is not intended to represent accurately the relative motion of different regions since there is no direct and simple relation between position along the $y$ axis and the $y$-projected velocity in such a perturbed outflow. Nonetheless, this 3D view offers valuable and complementary information to the 3D morphology of CIRs described above.

\section{Conclusion}

I have performed hydrodynamical simulations of line-driven winds subject to base variations taking the form of bright Gaussian spots. This investigation extended the work of Cranmer \& Owocki (1996) by moving the geometrical domain from $2 \mathrm{D}$ to $3 \mathrm{D}$. The radiative acceleration enhancement is no longer assumed purely radial but rather each of its three components is computed using a multi-ray quadrature of the visible stellar disk at every wind point.

I find that the general morphology of corotating interaction regions is unaffected by the spot latitude, and that indeed its key features are reproduced with the approach of $\mathrm{CO}$, i.e. which assumes that only the radial radiative acceleration is enhanced by the presence of bright spots. Thus, CIR properties seem to be dominated by the azimuthal modulation via stellar rotation of the perturbed radial radiative acceleration.

Nonetheless, the perturbed azimuthal velocity extrema are enhanced by a factor of about 50 following the explicit consideration of the lateral perturbed radiative acceleration. Although the exact value depends on the adopted spot amplitude, it appears that the dynamical effect of including the threecomponent multi-ray radiative acceleration could have implications for e.g. stars close to critical rotation for which material ahead of surface spots could be propelled with enough angular momentum to be maintained in orbit around the star (Owocki 2002; Owocki et al. 2004). This non-negligible lateral velocity field induces a "dynamical spreading" of the spot influence, weakening the CIR pattern.

The latitudinal extent of CIRs seems to be very well approximated by the full width at half maximum of the adopted spot, irrespective of its latitude. The latitude of advection of the CIR pattern is also that of the spot: CIRs are essentially contained within a conic shell whose opening angle is the colatitude of the spot and thickness the full-width-halfmaximum of the spot, a statement which applies to all adopted spot latitudes. This essentially radial nature of wind streamlines (for perturbed and unperturbed models) results from a combination of effects. Because of the constraint of angular 

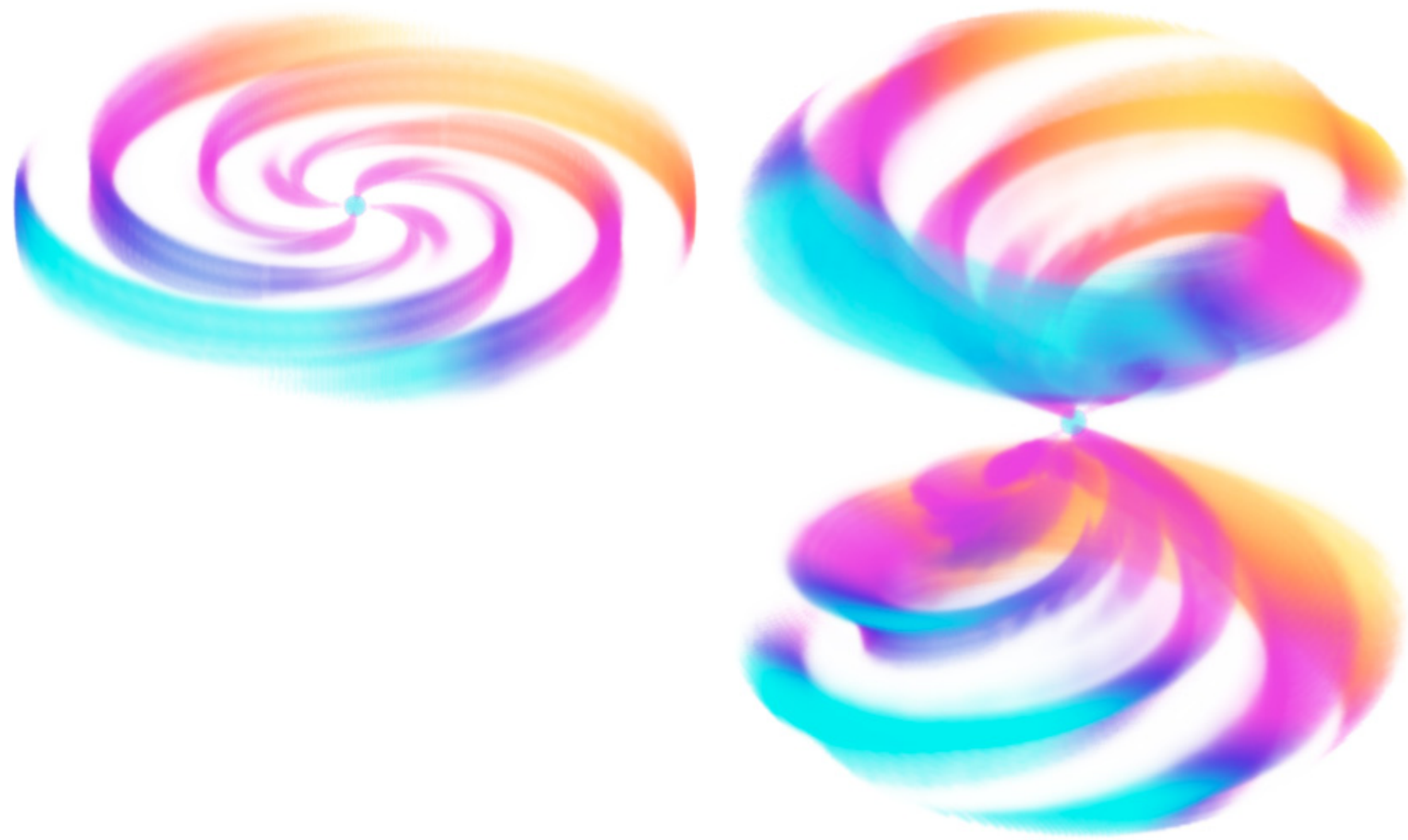

Fig. 7. 3D illustration based (this figure is available in color in electronic form) on a ray-tracing technique (Ruffert 1991) of the model described in Sect. 3.2 (left panel, spots in the equatorial plane) and Sect. 3.3 (right panel, spots at 45 deg latitude). The direction of the rotation axis is the vertical ( $z$ axis). The observer is placed at $1200 R_{\star}$ from the star centre and at an angle of 30 (left panel) and 20 deg (right panel) above the equatorial plane ( $x y)$ of the rotating star. The $x$ axis is to the right and the $y$ axis goes into the page so that (xyz) is direct. I choose a color-coding to differentiate between different wind regions along the $y$-axis. This is made more visible by applying a rotation of 30 deg of the observer with respect to the $y$ axis. For sake of clarity, the density contrast is artificially enhanced, showing only the density compression associated with the CIRs rather than the unperturbed medium in between them. This maximum allowed density contrast is fixed at three in the original dataset (rather than just above ten) and helps making the CIR appearance more uniform at all heights.

momentum conservation, wind material originating at offequatorial latitudes tends to experience an equatorward-drift as it advects. This is mitigated by the slight pole-ward tilt of the radiation force (arising from the higher radial velocity gradient in polar-tilted lines of sight) combined with the further reduction of azimuthal velocity resulting from the wind spin-down effect. Quantitatively, in my models, the latitudinal velocity field is found to be weak ( $15 \mathrm{~km} \mathrm{~s}^{-1}$ at most), hence negligible compared to the terminal velocity of the flow, i.e. $2600 \mathrm{~km} \mathrm{~s}^{-1}$ (Petrenz 1999; Petrenz \& Puls 2000). The focus of this study has been limited to slow rotators, but for objects closer to critical rotation, additional features might somewhat modify the current conclusions. The tendency for wind material to drift towards the equator will be more pronounced (being maximum for stars whose wind terminal velocity and rotational velocity are comparable), further favored, in the presence of gravity darkening, by the brighter polar regions that introduce an equatorward-tilt in the radiation force. However, the latitudinal component of the radiation force, in the presence of an oblate surface for such a near-critically rotating star, is expected to be strong enough to inhibit this equatorward drift and actually favor polar mass loss. Due to the strong non-linear coupling between all these different mechanisms, I will, in my next study, include the effects of oblateness to allow investigations for rotation speeds going from modest to near-critical values.

Limiting the discussion to slow rotators, one can ask what impact these changes in CIR properties will have on synthetic line profile calculations done so far in the same context $(\mathrm{CO}$, Dessart \& Chesneau 2002). To that end, I computed P-Cygni as well as emission line profiles for different line opacities using the technique described in $\mathrm{CO}$, based on the relevant hydrodynamical inputs (see Sect. 3.1). I did not consider off-equatorial spots since in that case lateral effects are similar or weaker than in the equatorial plane and because variability in P-Cygni troughs will occur through the presence of variations occurring in the column of material lying between the observer and the stellar disk, hence independent of spot location. Preliminary results indicate that no obvious effect based on distinct hydrodynamical inputs is discernable in P-Cygni and emission line profile variability. A wealth of variations can however be identified in the case of emission lines for differing inclination angles and spot latitudes. I defer till a later study the proper analysis of these spectroscopic variations and the comparison with observations.

Acknowledgements. I wish to thank Stan Owocki for his insightful comments and suggestions on a preliminary version of this 
article. All hydrodynamics simulations were performed on the basis of the ZEUS-3D code developed at the National Centre for Supercomputing Applications (NCSA) at the University of Urbana, Illinois.

\section{References}

Abbott, D. C. 1982, ApJ, 259, 282

Castor, J. I., Abbott, D. C., \& Klein, R. I. 1975, ApJ, 195, 157 (CAK)

Batchelor, G. K. 1967, An introduction to fluid dynamics (Cambridge: Cambridge University Press), 598

Bjorkman, J. E., \& Cassinelli, J. P. 1993, ApJ, 409, 429

Cranmer, S. R., \& Owocki, S. P. 1995, 440, 308

Cranmer, S. R., \& Owocki, S. P. 1996, ApJ, 462, 469

Dessart, L., \& Chesneau, O. 2002, A\&A, 395, 209

Dessart, L., \& Owocki, S. P. 2002a, A\&A, 383, 1113

Dessart, L., \& Owocki, S. P. 2002b, A\&A, 393, 991

Dessart, L., \& Owocki, S. P. 2003, A\&A, 406, L1

Eversberg, T., Lépine, S., \& Moffat, A. F. J. 1998, ApJ, 494, 799

Gayley, K. G., \& Owocki, S. P. 2000, ApJ, 537, 461

Humphreys, R. M., \& Davidson, K. 1994, PASP, 106, 1025
Hubeny, I., Heap, S. R., \& Altner, B. 1991, ApJ, 377, 33

Kaper, L., Henrichs, H. K., Fullerton, A. W., et al. 1997, A\&A, 327, 281

Koninx, J. P. M. 1992, Ph.D. dissertation, Rijksuniversiteit Utrecht

Lépine, S., \& Moffat, A. F. J. 1999, ApJ, 514, 909

Lucy, L. B., \& Solomon, P. 1970, ApJ, 159, 879

Owocki, S. P., \& Rybicki, G. B. 1984, ApJ, 284, 337

Owocki, S. P., Castor, J. I., \& Rybicki, G. B. 1988, ApJ, 335, 914

Owocki, S. P., Cranmer, S. R., \& Fullerton, A. W. 1997, ApJ, 453, 37

Owocki, S. P. 2002, in Stellar rotation, ed. A. Maeder, \& P. Eenens, ASP Conf. Ser., IAU Symp., in press

Petrenz, P. 1999, Ph.D. Thesis, Ludwig Maximiliam University Munich

Petrenz, P., \& Puls, J. 2000, A\&A, 358, 956

Prinja, R. K., Massa, D., \& Fullerton, A. W. 1995, ApJ, 452, 61

Robert, C. 1992, Ph.D. Thesis, Univ. Montréal

Ruffert, M. 1991, Int. J. Mod. Phys. C, 2, 1, 461

Rybicki, G. B., Owocki, S. P., \& Castor, J. I. 1990, ApJ, 349, 274

Stone, J. M., \& Norman, M. J. 1992, 80, 753 